\title{
Gemmeiza 12 - A NEW EGYPTIAN HIGH YIELDING AND RUST RESISTANT BREAD WHEAT (Triticum aestivum L.) CULTIVAR
}

Sadek ,Eman M.; A.Ageez; M.El-Menofy; M. Abo Shereef; A. Hamada; A.Moussa; R. Kumber; S. Sleem; G. El- Shaaray; A. A. morad; S. Abdel- Majeed; A. Abo- Warda; A.Tammam; M. Mesherf; E.A.M.El-Sayed; H. Ashoush; M. Toweefles; $H$. Hendawy;Hayam S. Mahgoub; A.K. Mostafa; H. El-Borhamy; A. Menshawy; Wafaa M. El- Awady Nadia Abd El-Nour; S. AbdelDayem; Sohair M. Hassan; A. Swelam; S. El- Sawy; S. Hamad;Magda A. Abdel - Rahman; Sabah. Abo El-Ela; M.A. Khaled; R.A. Ramadan; I. A. Amin; M. Zakaria; Manal A. Hassan; A. Gad-Allah; M. A. El-Maghraby; Aza M. Abdel-Al; A. Hagras; A.T. Mostafa; M.S. Mahmoud; M.Y.Moubark; Thanaa Abd-EIKreem; A.M.Morsy; Hoda El-Gharabawy; Aglan M.A.A; W. Farahat; Abd El-Hamid E.A.M;K.E.Ragab ;K.I.Gad;E; Nathan Shereen;Abdel- Latif, I.S.M; Abdel Kader,M.N; A. Gomaa ;Enayat Ghanem; S.Kh. Mahmoud; M.G.Mosaad; N.Hanna; M.A. Moussa; M.A.Gouda; M.A. Salem; A. Khattab; A. Abdel-Latif;A. El-Hag; Najwa Abdel- Fattah; F. Hefnawy ;Mostafa, M. A.; M.S. Sharshar; T. Shehab EI Din; M. Abde-Aleem; S.R.Sabry; M.S. Saleh; ${ }^{\star} I$. A. Imbaby and * $M$. A. Hasan.

Wheat Res. Dept. Field Crops Res. Inst., ARC, Giza 12619, Egypt.

*Wheat Disease Dept., Plant Pathology Res. Inst., ARC, Giza 12619, Egypt

\section{ABSTRACT}

Gemmeiza 12, a new bread wheat variety released by Wheat Research Department, Field Crops Research Institute, ARC, Egypt in 2012, offers wheat growers a new choice for high yielding, and rust resistant variety. Gemmeiza 12 originated and selected from a hybrid made at the International Maize and Wheat Improvement Center (CIMMYT). The parentages and pedigree of Gemmeiza 12 are : OTUS/3/SARA/THB//VEE CMSS97Y00227S-5y-010M-010Y-010M-2Y-1M-0Y-OGM .Based on the results of 76 yield trials conducted during three successive growing seasons $2007 / 2008,2008 / 2009$ and $2009 / 2010$ and 73 verification yield trials through the three growing seasons $2010 / 2011$ and 2011/2012 and 2012/2013 in addition to 22 demonstration fields during 2012/2013 growing season.

The results proved the superiority of the new cultivar Gemmeiza 12 over most of the dominating wheat cultivars i.e, Sakha 93, Gemmeiza 9, Giza 168 and Sids 12 at Delta region and Middle Egypt. Moreover, the new cultivar yielded more than Sakha 93 at all locations representing Upper Egypt and Out Valley zones but its productivity was slightly less than Giza 168 and Sids 12 in both locations. The results obtained from the on-farm trials and the demonstration field assure the superiority of Gemmeiza 12 The new released variety was resistant to rust diseases especially both yellow and stem rust diseases. The flour of Gemmeiza 12 is acceptable for bread making since the protein content reached about $13 \%$ and the wet gluten percentage was $29.1 \%$ 


\section{INTRODUCTION}

Wheat (Triticum aestivum L.), the cereal leading crop is the main human staple food in Egypt as well as in many countries over all the world. Moreover, it is considered to be the major source of straw fodder for animal feeding. The Egyptian national wheat production in 2012/2013 season approximately amounted 9.46 million ton while the total consumption was about 16 million tons of grains and flour. Therefore, there is a need to increase the total wheat production to overcome the gap between production and consumption.

In the short term, it is not feasible to increase the area devoted to wheat. Increased production per unit area appears to be the main possible mean of reducing the wheat gap.The required yield increases may be achieved by introducing high yielding varieties and simultaneously implementing improved cultural practices. Such improved varieties must resist or tolerate the unfavorable environments and be stable in a broad spectrum of environments.

Egypt's national wheat research program adopted the strategy of Comstock and Moll (1963) to stabilize wheat production, by separating environments by macro- environmental differences such as temperature, rainfall and soil type and developing several varieties, each highly adapted to a closely identified sub-region with a relatively uniform environment under this condition, trials usually show a small variance due to genotype environment interaction, with a major variance due to genetics. Current policy favors use of more than one cultivar in a given location, therefore, more varieties with wide adaptability one sought to improve yield performance and stabilize production. However, developing narrowly adapted or location specific varieties suited to specific zones or locations must not be ignored.

Wheat yield have increased gradually over the past years, from 4.95 $\mathrm{ard} / \mathrm{fed}$ in the year 1950 to $18.67 \mathrm{ard} / \mathrm{fed}$ in the year 2013.In a study by ( Ageez et.al., 1999 )came to a conclusion that the genetic yield increase amounted $4.2 \%$ per year during 50 years period from 1950 till the year 2000 .

To achieve yield maximization and avoid a plateau or decline in average yields, a continuous efforts and effective breeding program are essential. Ongoing efforts must be focused and subjecting promising selected materials to yield and agronomic evaluation in more sites to obtain reliable data and use off-station testing for yield verification and demonstration in framer's fields.

The national wheat research program had released the two varieties Sakha 61 for Delta region and Sakha 69 for all over the country due to its wide adaptability (Gomma et. al., 1984).Moreover, more wheat cultivars were released during nineties namely, Gemmeiza 3( El-Sayed et.al., 1996), Gemmeiza 5 (Mitkees et.al., 1998), Sakha 93 and Giza 168 which were characterized by their high resistance to strip rust and high yielding ability (Shehab El- Din et.al., 1999) in addition to the wheat cultivar Gemmeiza 9 which proved its superiority on the dominating wheat cultivars Sakha 93, Sids 1 and Gemmeiza 5 as well as its highly resistance to rust diseases (Mosaad et. al., 2000) 
The Egyptian wheat breeders through their continuous efforts had released further bread wheat cultivars, i.e. Gemmeiza 10 (El- Shami et. al. 2005), Sids 12 (Mahrous et. al., 2009), Sids 13 Moustafsa et. al., 2010) and Gemmeiza 11(Sadek, Eman et. al., 2013).

The present research work was undertaken to introduce a new Egyptian wheat cultivar resistant to rust diseases and high yielding ability under different environmental conditions.

\section{MATERIALS AND METHODS}

The new bread wheat cultivar Gemmeiza 12 has been selected from CIMMYT materials grown at El- Gemmeiza Agricultural Research Station, ARC. in 2005/2006 season. The cross name and pedigree of the new cultivar is OTUS/3/SARA/THB//VEE (CMSS97YOO227 S-5Y-010M-010Y- 010M-2Y - 1M-OY-OGM). Moreover, the following yield trials were carried out to test the performance of the new wheat cultivar Gemmeiza 12 as follows:

\section{1-Preliminary yield trials:}

The newly released cultivar Gemmeiza 12 was advanced to Gemmeiza screening nurseries in 2006/2007 season. Gemmeiza 12 was tested for grain yield ability in 2007/2008 growing season against 64 promising lines including the dominating local wheat cultivars Sakha 93, Gemmeiza 9, Giza 168 and Sids 12 in 9 preliminary yield trials conducted at nine research stations representing different ecological zones of wheat growing regions at over all the country ;i.e. North Delta, Middle Delta , Middle Egypt , Upper Egypt ,and Out Valley .The experimental design used in these trials was randomized complete block design (RCBD) with four replicates according to Steel and Torrie (1960). The plot area was $4.8 \mathrm{~m}^{2}$ including 6 rows , $4 \mathrm{~m}$ long and 20 $\mathrm{cm}$ apart.

\section{2- Advanced yield trials:}

In the next season 2008/2009, the new cultivar Gemmeiza 12 and 15 other promising lines including four local check cultivars , Sakha 93 , Gemmeiza 9, Giza 168 and Sids 12 were tested for yielding ability in 29 advanced yield trials in different ecological zones. Again the new cultivar was tested in 2009/2010 growing season along with 16 promising genotypes including the check cultivars Sakha 93, Gemmeiza 9 and Giza 168 in the second phase of advanced yield trials. In these yield trials, all promising lines as well as the check cultivars were tested in large plot area experiment (3.0 $\times 3.5 \mathrm{~m}=10.5 \mathrm{~m}^{2}$ each) using planting method usually the farmers used (broadcasting). In addition, five advanced yield trials in both seasons including the same genotypes were conducted using drill planting method only at the main Agricultural Research Stations i.e., Gemmeiza, Sakha, Giza, Sids and Shandaweel. The statistical design was RCBD with four replications according to Steel and Torrie (1960).

\section{3- Verification yield trials:}

Seventy-Three on-farm trials were carried out in the farmer's fields in the old and new lands representing over all Egypt in three successive

$$
19.1
$$


seasons 2010/2011, 2011/2012 and 2012/2013 including the new released cultivar and the dominating wheat cultivars i.e., Sakha 93, Gemmeiza 9, Giza 168 and Sids 12 .The area of each experimental plot was $\left(100 \mathrm{~m}^{2}\right)$ for each cultivar. Four randomly selected samples $4 \mathrm{~m}^{2}$ were harvested and grain yield was weighted and adjusted to ard/fed.

\section{4- Demonstration fields:}

Twenty Two demonstration fields including Gemmeiza 12 along with 4 dominating wheat cultivars were carried out at 4 governorates representing Delta region. The area of each selected field was $1 \mathrm{fed}$. The demonstration field was planted in hills $10 \mathrm{~cm}$ apart on raised beds and the width of beds was $120 \mathrm{~cm}$. At harvesting time, 4 randomly selected samples $\left(4 \mathrm{~m}^{2}\right)$ were harvested and threshed.The grain yield of each sample was weighted and adjusted to $\mathrm{ard} / \mathrm{fed}$.

\section{5- Disease severity:}

Disease severity score, expressed as the \% coverage of leaves with rust pustules and plant reaction, to indicate the infection type; $0=$ immune , $R=$ resistant, $M R=$ moderately resistant, $M S=$ moderately susceptible and $S=$ Susceptible were recorded. Moreover, Average Coefficient of Infection (ACI) was calculated using adapted scale of Saari and Wilcoxson (1974) as follows: $\mathrm{O}=0.0, \mathrm{R}=0.2, \mathrm{MR}=0.4, \mathrm{X}$ (mixed reaction) $=0.6, \mathrm{MS}=0.8$ and $\mathrm{S}=1.0$

\section{6- Technological and quality characters:}

Some technological and quality characters i.e, protein \%, ash \%, extraction rate \% and gluten \% (wet and dry) for Gemmeiza 12 in comparison with 4 dominating wheat cultivars were done by Crop Technology Research Department, Food Technology Research Institute, ARC.

\section{7- Distinctness Uniformity and Stability tests (DUS):}

The morphological characteristics of Gemmeiza 12 were done by the Central Administration of Seed Certification (CASC) according to the International Union for the Protection of new Varieties of plants (UPOV).

\section{RESULTS AND DISCUSSION}

\section{1- Preliminary yield trials:}

The results given in ( Table 1-a) showed the grain yield (ard/fed) of the new released bread wheat Gemmeiza 12 and four commercial wheat cultivars in 2007/2008 growing season at Delta region. The new cultivar yielded almost the same as Sakha 93 , Gemmeiza 9 and Giza 168 and significantly at over all locations less than Sids 12 wheat cultivar .Meanwhile, the yield of Gemmeiza 12 yielded about 2.1\% less than the checks mean at Delta region i.e. Sakha ,Gemmeiza and Kafr El-Hamam Research Stations. Grain yield of the new cultivar exceeded all the commercials wheat cultivars mean at Middle Egypt by $6.7 \%$ (Table 1-b).Likewise, the new cultivar Gemmeiza 12 outyielded all check cultivars at Upper Egypt with significant increase over Giza 168 and Sids 12.Moreover, the grain yield increase of 
Gemmeiza 12 was only $0.6 \%$ over the checks mean at El-Nubaria Research Station. (Table 1- c). Results of the preliminary yield trial at overall Egypt indicate that the new wheat cultivar Gemmeiza 12 out yielded the checks mean, Sakha 93, Giza 168 and Gemmeiza 9 while, yielded the same as Sids 12 (Table 1-d).

Table (1-a):Grain yield (ard./fed.) of the preliminary yield trials for Gemmeiza 12 and four bread wheat cultivars at Delta region in 2007/2008 season.

\begin{tabular}{|l|c|c|c|c|}
\hline \multirow{2}{*}{ Cultivars } & \multicolumn{3}{|c|}{ Locations } & \multirow{2}{*}{ Mean } \\
\cline { 2 - 4 } & Sakha & El-Gemmeiza & $\begin{array}{c}\text { Kafer El- } \\
\text { Hamam }\end{array}$ & \\
\hline Sakha 93 & 22.71 & 25.21 & 16.37 & 21.43 \\
\hline Gemmeiza 9 & 20.40 & 26.96 & 19.78 & 22.38 \\
\hline Giza168 & 23.42 & 24.12 & 19.25 & 22.26 \\
\hline Sids 12 & 23.74 & 27.10 & 20.11 & 23.56 \\
\hline Checks mean & 22.50 & 25.85 & 18.88 & 22.41 \\
\hline Gemmeiza 12 & $\mathbf{2 2 . 0 7}$ & $\mathbf{2 4 . 0 6}$ & $\mathbf{1 9 . 6 9}$ & $\mathbf{2 1 . 9 4}$ \\
\hline C.V\% & 8.89 & 8.02 & 8.79 & 8.59 \\
\hline L.S.D. at 5\% & 2.71 & 2.76 & 2.33 & 2.60 \\
\hline
\end{tabular}

Table (1-b):Grain yield (ard./fed.) of the preliminary yield trials for Gemmeiza 12 and four bread wheat cultivars at Middle Egypt in $2007 / 2008$ season.

\begin{tabular}{|l|c|c|c|c|}
\hline \multirow{2}{*}{ Cultivars } & \multicolumn{3}{|c|}{ Locations } & \multirow{2}{*}{ Mean } \\
\cline { 2 - 4 } & Giza & Sids & Mallawy & \\
\hline Sakha 93 & 14.17 & 25.05 & 19.97 & 19.73 \\
\hline Gemmeiza 9 & 12.42 & 21.39 & 19.61 & 17.81 \\
\hline Giza 168 & 12.83 & 20.28 & 21.78 & 18.30 \\
\hline Sids 12 & 9.25 & 23.71 & 23.49 & 18.82 \\
\hline Checks mean & 12.17 & 22.61 & 21.21 & 18.66 \\
\hline Gemmeiza 12 & $\mathbf{1 2 . 4 2}$ & $\mathbf{2 5 . 9 2}$ & $\mathbf{2 1 . 4 1}$ & $\mathbf{1 9 . 9 1}$ \\
\hline C.V\% & 25.59 & 10.00 & 4.67 & 13.42 \\
\hline L.S.D. at 5\% & 2.21 & 3.08 & 1.33 & 2.21 \\
\hline
\end{tabular}

Table (1-c): Grain yield (ard./fed.) of the preliminary yield trials for Gemmeiza 12 and four bread wheat cultivars at Upper Egypt and Out Valley in 2007/2008 season.

\begin{tabular}{|l|c|c|c|c|}
\hline \multirow{2}{*}{ Cultivars } & \multicolumn{2}{|c|}{ Locations } & \multirow{2}{*}{ Mean } & Out Valley \\
\cline { 2 - 3 } \cline { 5 - 5 } & Shandaweel & Matanaa & & Nubaria \\
\hline Sakha 93 & 22.25 & 23.79 & 23.02 & 12.47 \\
\hline Gemmeiza 9 & 22.95 & 21.98 & 22.47 & 16.14 \\
\hline Giza 168 & 20.93 & 19.80 & 20.37 & 16.60 \\
\hline Sids 12 & 23.07 & 20.78 & 21.93 & 18.29 \\
\hline Checks mean & 22.30 & 21.59 & 21.95 & 15.88 \\
\hline Gemmeiza 12 & $\mathbf{2 3 . 9 4}$ & $\mathbf{2 5 . 4 3}$ & $\mathbf{2 4 . 6 8}$ & $\mathbf{1 5 . 9 7}$ \\
\hline C.V\% & 14.21 & 10.20 & 12.34 & 11.60 \\
\hline L.S.D. at 5\% & 4.42 & 3.19 & 2.73 & 2.66 \\
\hline
\end{tabular}


Table (1-d): Grain yield (ard./ fed.) of the preliminary yield trials for Gemmeiza 12 and four bread wheat cultivars at over all Egypt in 2007/2008 season.

\begin{tabular}{|l|c|c|c|c|c|}
\hline \multirow{2}{*}{ Cultivars } & \multicolumn{4}{|c|}{ Locations } & \multirow{2}{*}{ Mean } \\
\cline { 2 - 5 } & Delta & $\begin{array}{c}\text { Middle } \\
\text { Egypt }\end{array}$ & $\begin{array}{c}\text { Upper } \\
\text { Egypt }\end{array}$ & Out valley & \\
\hline Sakha 93 & 21.43 & 19.73 & 23.02 & 12.47 & 19.16 \\
\hline Gemmeiza 9 & 22.83 & 17.81 & 22.47 & 16.14 & 19.81 \\
\hline Giza 168 & 22.26 & 18.30 & 20.37 & 16.60 & 19.38 \\
\hline Sids 12 & 23.56 & 18.82 & 21.93 & 18.29 & 20.65 \\
\hline Checks mean & 22.41 & 18.66 & 21.95 & 15.88 & 19.73 \\
\hline Gemmeiza 12 & $\mathbf{2 1 . 9 4}$ & $\mathbf{1 9 . 9 1}$ & $\mathbf{2 4 . 6 8}$ & $\mathbf{1 5 . 9 7}$ & $\mathbf{2 0 . 6 3}$ \\
\hline C.V\% & 8.59 & 14.79 & 12.34 & 11.60 & 11.82 \\
\hline L.S.D. at 5\% & 1.50 & 2.21 & 2.73 & 2.66 & 2.28 \\
\hline
\end{tabular}

\section{2-Advanced yield trials:}

Data in (Table 2-a) show the grain yield of advanced yield trials for Gemmeiza 12 and four bread wheat cultivars at North Delta in 2008/2009 growing season. The newly bread wheat cultivar Gemmeiza 12 gave more grain yield than the check cultivars Sakha 93 , Giza 168 and Sids 12 but less grain yield than Gemmeiza 9 with an average increase of $3.9 \%$ over the checks mean at North Delta.

The new released cultivar Gemmeiza 12 yielded more than all tested wheat cultivars at Gemmeiza, Kafr El-Hamam and Sharkia-2 locations at South Delta region. The grain yield of the new cultivar exceeded all the commercial wheat cultivars i.e.Sakha 93, Gemmeiza 9, Giza 168 and Sids 12 with $4.5 \%$ increase over all the checks mean at South Delta region(Table2-b).

For the results of Middle Egypt region (Table 2-c), data showed that the yield of the newly bread wheat cultivar had overcome the checks cultivars Sakha 93, Gemmeiza 9 and Giza 168 at over all locations while, yielded almost the same as Sids 12 cultivar. The increase in grain yield over checks mean was estimated by $4.3 \%$ at Middle Egypt.

The results of advanced yield trials at Upper Egypt (Table 2-d) in 2008/2009 season cleared that the new released wheat cultivar Gemmeiza 12 yielded $23.35 \mathrm{ard} / \mathrm{fed}$ almost the same as the check cultivar Gemmeiza 9 and surpassed the other three checks Sakha 93, Giza 168 and Sids 12 and the checks mean at Shandaweel location .Meanwhile, its productivity was significantly less than the check cultivars at El-Matanaa location while, yielded almost the same as Sakha 93, Gemmeiza 9 and Giza 168 and significantly less than Sids 12 at Kom-Ombo .The productivity of the new cultivar at over all Upper Egypt was 6.3\% less than the checks mean.

Data in Table 2-e revealed that Gemmeiza 12 yielded more than all check cultivars at Gemmeiza, Giza and Sids Research Stations when using drill planting method .Meanwhile, at Sakha research station the yield of the new cultivar surpassed Sakha 93, Gemmeiza 9 and Giza 168. The yield of the new released cultivar surpassed the checks mean at the five Agricultural Research Stations by $10.8 \%$ when using drill planting method. 
The data presented in Table 2-f showed that the new cultivar was less in its yield productivity compared with the local checks at all Out Valley region. The yield productivity was estimated by about $3.8 \%$ less than all checks mean at over all Out Valley locations.

The results of the advanced trials over all Egypt in 2008/2009 season (Table 2-j) cleared that the new cultivar Gemmeiza 12 outyielded the check cultivar Sakha 93 and Giza 168 at North and South Delta, Middle Egypt and when using drill planting method in addition to its superiority over the check cultivar Sids 12 at North and South Delta and in drill sowing method. The average increase in grain yield of Gemmeiza 12 above all checks mean at over all Egypt in 2008/2009 season was only $2.7 \%$ although its yield productivity was insignificantly less than the check cultivars Giza 168 and Sids 12 at both Upper Egypt and Out Valley regions.

Table(2-a): Grain yield (ard/ fed.) of the advanced yield trials (D.BW)for Gemmeiza 12 and four bread wheat cultivars at North Delta in $2008 / 2009$ season.

\begin{tabular}{|c|c|c|c|c|c|c|c|}
\hline \multirow[t]{2}{*}{ Cultivars } & \multicolumn{6}{|c|}{ Locations } & \multirow[b]{2}{*}{ Mean } \\
\hline & $\begin{array}{c}\text { El- } \\
\text { Serw }\end{array}$ & $\begin{array}{c}\text { Dakhalia } \\
1\end{array}$ & \begin{tabular}{|} 
Dakhalia \\
2
\end{tabular} & Sakha & $\begin{array}{c}\text { Etai- } \\
\text { Elbarood }\end{array}$ & Behiraa & \\
\hline Sakha 93 & 20.23 & 19.01 & 10.47 & 25.73 & 18.07 & 21.33 & 19.14 \\
\hline Gemmeiza 9 & 18.09 & 20.58 & 15.40 & 26.81 & 21.90 & 24.67 & 21.24 \\
\hline Giza 168 & 18.61 & 21.52 & 13.87 & 26.82 & 19.07 & 22.03 & 20.31 \\
\hline Sids 12 & 16.73 & 24.98 & 12.20 & 27.41 & 14.53 & 18.67 & 19.09 \\
\hline Checks mean & 18.42 & 21.52 & 12.99 & 26.69 & 18.39 & 21.67 & 19.95 \\
\hline Gemmeiza 12 & 20.16 & 23.97 & 14.67 & 28.33 & 16.60 & 20.67 & 20.73 \\
\hline C.V\% & 10.91 & 3.65 & 5.72 & 6.07 & 5.39 & 7.40 & 6.52 \\
\hline L.S.D. at $5 \%$ & 2.78 & 1.09 & 1.10 & 2.34 & 1.35 & 2.15 & 1.86 \\
\hline
\end{tabular}

Table (2-b): Grain yield (ard / fed.) of the advanced yield trials (D-BW) for Gemmeiza 12 and four bread wheat cultivars at South Delta in 2008/2009 season.

\begin{tabular}{|c|c|c|c|c|c|c|c|c|c|}
\hline \multirow[b]{2}{*}{ Cultivars } & \multicolumn{8}{|c|}{ Locations } & \multirow[b]{2}{*}{ Mean } \\
\hline & Gemmeiza & $\begin{array}{c}\text { Sers } \\
\text { El-Liaan }\end{array}$ & $\begin{array}{l}\text { Tag } \\
\text { El-Ez }\end{array}$ & $\begin{array}{c}\text { Kafr } \\
\text { El- } \\
\text { Hamam }\end{array}$ & $\begin{array}{c}\text { Sharkia } \\
-1\end{array}$ & $\begin{array}{c}\text { Sharkia } \\
-2\end{array}$ & Monufia & Qaluobia & \\
\hline Sakha 93 & 22.63 & 23.47 & 17.90 & 21.33 & 21.23 & 18.23 & 22.27 & 28.13 & 21.9 \\
\hline Gemmeiza9 & 24.13 & 23.50 & 17.58 & 21.38 & 23.70 & 16.90 & 23.40 & 32.00 & 22.82 \\
\hline Giza 168 & 23.47 & 25.57 & 17.67 & 22.73 & 23.73 & 18.70 & 24.00 & 29.67 & 23.19 \\
\hline Sids 12 & 23.20 & 24.50 & 23.10 & 21.25 & 23.53 & 17.07 & 20.20 & 28.53 & 22.67 \\
\hline Checks mean & 23.36 & 24.26 & 19.06 & 21.67 & 23.05 & 17.73 & 22.47 & 29.58 & 22.65 \\
\hline Gemmeiza 12 & 26.27 & 24.93 & 21.59 & 24.91 & 20.23 & 19.90 & 22.67 & 28.93 & 23.68 \\
\hline C.V \% & 7.07 & 9.44 & 4.59 & 2.58 & 7.33 & 9.08 & 16.88 & 11.94 & 8.61 \\
\hline L.S.D. at $5 \%$ & 2.39 & 3.26 & 1.20 & 0.83 & 2.32 & 2.24 & 5.57 & 4.95 & 2.85 \\
\hline
\end{tabular}

In 2009/2010 growing season, yields of the new variety Gemmeiza 12 and the common check cultivars Sakha 93, Gemmeiza 9 and Giza 168 are given in Tables 3a, b,c,d,e,f and j. In North Delta (Table 3-a) , Gemmeiza 12 gave more grain yield than Sakha 93 , Giza 168 and the checks mean at ElSerw, Dakhalia 1, Dakhalia 2 and Sakha locations in addition to Sakha 93 at

$$
17.0
$$


Etai- Elbarood location. Meanwhile, the productivity of the new cultivar was significantly less than Sakha 93 and insignificantly less than Gemmeiza 9 and Giza 168 at El-Behiraa location.The grain yield of the new variety exceeded the checks mean by $2.97 \%$ at North Delta region.

Table (2-c): Grain yield (ard / fed.) of the advanced yield trials (D-BW) for Gemmeiza 12 and four bread wheat cultivars at Middle Egypt in 2008/2009season.

\begin{tabular}{|l|c|c|c|c|c|c|}
\hline \multirow{2}{*}{ Cultivars } & \multicolumn{7}{|c|}{ Locations } \\
\cline { 2 - 7 } & Giza & Fayoum & Sids & Mallawy & Menia & Mean \\
\hline Sakha 93 & 25.45 & 26.00 & 31.67 & 23.53 & 21.53 & 25.64 \\
\hline Gemmeiza 9 & 26.38 & 22.33 & 29.8 & 24.43 & 21.93 & 24.97 \\
\hline Giza 168 & 26.96 & 25.00 & 29.63 & 24.23 & 19.87 & 25.14 \\
\hline Sids 12 & 28.36 & 27.00 & 28.87 & 24.41 & 28.93 & 27.51 \\
\hline Checks mean & 26.79 & 25.08 & 29.99 & 24.15 & 23.07 & 25.82 \\
\hline Gemmeiza 12 & $\mathbf{2 8 . 7 1}$ & $\mathbf{2 4 . 0 0}$ & $\mathbf{3 0 . 7 3}$ & $\mathbf{2 3 . 2 3}$ & $\mathbf{2 7 . 9 3}$ & $\mathbf{2 6 . 9 2}$ \\
\hline C.V\% & 6.33 & 12.12 & 5.74 & 6.85 & 7.91 & 7.79 \\
\hline L.S.D. at 5\% & 2.41 & 4.19 & 2.48 & 2.32 & 2.41 & 2.76 \\
\hline
\end{tabular}

Table (2-d): Grain yield (ard / fed.) of the advanced yield trials (D-B.W) for Gemmeiza 12 and four bread wheat cultivars at Upper Egypt in 2008/2009 season.

\begin{tabular}{|l|c|c|c|c|}
\hline \multirow{2}{*}{ Cultivars } & \multicolumn{4}{|c|}{ Locations } \\
\cline { 2 - 5 } & Shandaweel & Mattana & Kom-Ombo & Mean \\
\hline Sakha 93 & 18.62 & 22.93 & 21.20 & 20.92 \\
\hline Gemmeiza 9 & 23.83 & 23.67 & 20.93 & 22.81 \\
\hline Giza 168 & 22.32 & 25.53 & 21.00 & 22.95 \\
\hline Sids 12 & 22.32 & 24.27 & 23.67 & 23.42 \\
\hline Checks mean & 21.78 & 24.10 & 21.70 & 22.52 \\
\hline Gemmeiza 12 & $\mathbf{2 3 . 3 5}$ & $\mathbf{1 9 . 4 0}$ & $\mathbf{2 0 . 6 0}$ & $\mathbf{2 1 . 1 2}$ \\
\hline C.V \% & 11.13 & 7.19 & 6.41 & 8.24 \\
\hline L.S.D. at 5\% & 3.42 & 2.35 & 1.96 & 2.58 \\
\hline
\end{tabular}

Table (2- e): Grain yield (ard / fed.) of the advanced yield trials (D-BW)Drill for Gemmeiza 12 and four bread wheat cultivars in 2008/2009 season

\begin{tabular}{|l|c|c|c|c|c|c|}
\hline \multirow{2}{*}{ Cultivars } & \multicolumn{7}{|c|}{ Locations } \\
\cline { 2 - 7 } & Gemmeiza & Sakha & Giza & Sids & Shandaweel & Mean \\
\hline Sakha 93 & 23.03 & 23.20 & 28.00 & 34.80 & 22.09 & 26.22 \\
\hline Gemmeiza 9 & 25.87 & 22.97 & 25.75 & 33.50 & 21.05 & 25.83 \\
\hline Giza 168 & 22.57 & 23.38 & 20.25 & 34.30 & 25.19 & 25.14 \\
\hline Sids 12 & 22.60 & 27.05 & 23.84 & 34.27 & 24.55 & 26.46 \\
\hline Checks mean & 23.52 & 24.15 & 24.46 & 34.22 & 23.22 & 25.91 \\
\hline Gemmeiza 12 & $\mathbf{2 9 . 4 3}$ & $\mathbf{2 5 . 6 8}$ & $\mathbf{3 0 . 3 3}$ & $\mathbf{3 6 . 2 3}$ & $\mathbf{2 1 . 9 3}$ & $\mathbf{2 8 . 7 2}$ \\
\hline C.V\% & 7.20 & 6.89 & 10.79 & 4.02 & 9.98 & 7.78 \\
\hline L.S.D. at 5\% & 2.58 & 2.42 & 3.81 & 1.99 & 3.29 & 2.82 \\
\hline
\end{tabular}


Table (2-f): Grain yield (ard / fed.) of the advanced yield trials (D-BW) for Gemmeiza 12 and four bread wheat cultivars at out Valley in 2008/2009 season.

\begin{tabular}{|l|c|c|c|c|c|c|c|}
\hline Cultivars & \multicolumn{7}{|c|}{ Locations } \\
\cline { 2 - 8 } & Nubaria & $\begin{array}{c}\text { Al- } \\
\text { Bostan }\end{array}$ & Assuit & $\begin{array}{c}\text { New } \\
\text { valley }\end{array}$ & Tushki & $\begin{array}{c}\text { El- } \\
\text { Ewinat }\end{array}$ & Mean \\
\hline Sakha 93 & 19.99 & 10.95 & 10.40 & 19.63 & 19.63 & 18.77 & 16.56 \\
\hline Gemmeiza 9 & 17.87 & 10.25 & 11.33 & 19.00 & 19.22 & 14.87 & 15.42 \\
\hline Giza 168 & 18.53 & 13.00 & 9.33 & 19.09 & 19.49 & 19.80 & 16.54 \\
\hline Sids 12 & 21.20 & 11.80 & 11.33 & 18.57 & 22.07 & 20.37 & 17.56 \\
\hline Checks mean & 19.40 & 11.50 & 10.60 & 19.07 & 20.10 & 18.45 & 16.52 \\
\hline Gemmeiza 12 & $\mathbf{1 4 . 6 0}$ & $\mathbf{1 1 . 5 2}$ & $\mathbf{1 1 . 3 3}$ & $\mathbf{1 8 . 8 4}$ & $\mathbf{1 8 . 7 9}$ & $\mathbf{2 0 . 2 3}$ & $\mathbf{1 5 . 8 9}$ \\
\hline C.V\% & 7.93 & 11.84 & 15.68 & 8.83 & 6.64 & 4.27 & 9.20 \\
\hline L.S.D. at 5\% & 1.76 & 1.95 & 2.33 & 2.58 & 1.86 & 1.16 & 1.94 \\
\hline
\end{tabular}

Table (2-j): Grain yield (ard / fed.) of the advanced yield trials (D-BW) for Gemmeiza 12 and four bread wheat cultivars at over all Egypt in 2008/2009 season.

\begin{tabular}{|c|c|c|c|c|c|c|c|}
\hline \multirow[b]{2}{*}{ Cultivars } & \multicolumn{6}{|c|}{ Locations } & \multirow[b]{2}{*}{ Mean } \\
\hline & $\begin{array}{l}\text { North } \\
\text { Delta }\end{array}$ & $\begin{array}{l}\text { South } \\
\text { Delta }\end{array}$ & $\begin{array}{c}\text { Middle } \\
\text { Egypt }\end{array}$ & $\begin{array}{l}\text { Upper } \\
\text { Egypt }\end{array}$ & $\begin{array}{c}\text { Out } \\
\text { Valley }\end{array}$ & Drill & \\
\hline Sakha 93 & 19.14 & 21.9 & 25.64 & 20.92 & 16.56 & 26.22 & 21.73 \\
\hline Gemmeiza 9 & 21.24 & 22.82 & 24.97 & 22.81 & 15.42 & 25.83 & 22.18 \\
\hline Giza 168 & 20.31 & 23.19 & 25.14 & 22.95 & 16.54 & 25.14 & 22.21 \\
\hline Sids 12 & 19.09 & 22.67 & 27.51 & 23.42 & 17.56 & 26.46 & 22.79 \\
\hline Checks mean & 19.95 & 22.65 & 25.82 & 22.53 & 16.52 & 25.91 & 22.23 \\
\hline Gemmeiza 12 & 20.73 & 23.68 & 26.92 & 21.12 & 15.89 & 28.72 & 22.84 \\
\hline C.V \% & 6.52 & 8.61 & 7.79 & 8.24 & 9.20 & 7.78 & 8.02 \\
\hline L.S.D. at $5 \%$ & 1.81 & 2.84 & 2.76 & 2.57 & 1.94 & 2.82 & 2.45 \\
\hline
\end{tabular}

The new wheat cultivar Gemmeiza 12 yielded $20.76 \mathrm{ard} / \mathrm{fed}$ at over all mean of nine locations representing South Delta region. The estimated increase was $3.5 \%$ over the checks mean (Table 3-b). Moreover, at Middle Egypt, the yield of the new bread wheat cultivar Gemmeiza 12 exceeded the check cultivars at all the tested locations in Middle Delta region. The average increase in grain yield of Gemmeiza 12 above all checks mean cultivars at over all Middle Egypt was 7.3\% (Table 3-c).

Regarding to Upper Egypt, (Table 3-d), the new cultivar Gemmeiza 12 yielded insignificantly less than all check cultivars at Shandaweel, Gemmeiza 9 at El-Mattanaa and both Gemmeiza 9 and Giza 168 at KomOmbo. The decrease in grain yield at over all Upper Egypt regions reached about $-3.1 \%$.

The grain yield (ard/fed) of the advanced yield trials when sown by drill planting method for Gemmeiza 12 and three bread wheat cultivars in 2009/2010 growing season are presented in Table 3-e .The new cultivar proved its superiority above Gemmeiza 9 and Giza 168 cultivars at Gemmeiza, Sids,Sakha and Shandaweel locations while, yielded more over Sakha 93 at Giza location. The average yield increase of Gemmeiza 12 was 
$1.13 \mathrm{ard} / \mathrm{fed}(4.8 \%)$ when compared with the means of all check cultivars across all tested locations.

Table (3-a): Grain yield (ard / fed.) of the advanced yield trials (D-BW )for Gemmeiza 12 and three bread wheat cultivars at North Delta in 2009/2010 season.

\begin{tabular}{|l|c|c|c|c|c|c|c|}
\hline \multirow{2}{*}{ Cultivars } & \multicolumn{7}{|c|}{ Locations } \\
\cline { 2 - 8 } & $\begin{array}{c}\text { El- } \\
\text { Serw }\end{array}$ & Dakahlia 1 & Dakahlia 2 & Sakha & Etai-Elbarood & Behira & Mean \\
\hline Sakha 93 & 16.82 & 9.54 & 10.40 & 20.49 & 13.27 & 23.13 & 15.60 \\
\hline Gemmeiza 9 & 16.28 & 13.47 & 13.49 & 21.25 & 18.67 & 20.53 & 17.28 \\
\hline Giza 168 & 16.83 & 10.8 & 12.27 & 21.47 & 17.54 & 21.13 & 16.67 \\
\hline Checks mean & 16.63 & 11.27 & 12.05 & 21.07 & 16.49 & 21.6 & 16.52 \\
\hline Gemmeiza 12 & $\mathbf{1 8 . 9 0}$ & $\mathbf{1 1 . 8 0}$ & $\mathbf{1 2 . 6 0}$ & $\mathbf{2 3 . 1 1}$ & $\mathbf{1 6 . 1 4}$ & $\mathbf{1 9 . 5 0}$ & $\mathbf{1 7 . 0 1}$ \\
\hline C.V \% & 7.42 & 21.36 & 21.66 & 8.04 & 5.19 & 5.97 & 11.61 \\
\hline L.S.D. at 5\% & 1.82 & 3.53 & 3.85 & 2.54 & 1.25 & 1.83 & 2.47 \\
\hline
\end{tabular}

Table (3-b): Grain yield (ard / fed.) of the advanced yield trials (D-BW) for Gemmeiza 12 and three bread wheat cultivars at South Delta in 2009/2010 season.

\begin{tabular}{|c|c|c|c|c|c|c|c|c|c|c|}
\hline \multirow{2}{*}{ Cultivars } & \multicolumn{7}{|c|}{ Locations } & \multirow[b]{2}{*}{ Qaluobia } & \multirow[b]{2}{*}{ Bahteem } & \multirow{2}{*}{ Mean } \\
\hline & Gemmeiza & \begin{tabular}{|c|} 
Sers \\
El-lian
\end{tabular} & $\begin{array}{l}\text { Tag } \\
\text { El-Ez }\end{array}$ & \begin{tabular}{|l|} 
Kafr El- \\
Hamam
\end{tabular} & $\mid \begin{array}{c}\text { Sharkia } \\
1\end{array}$ & \begin{tabular}{|c|} 
Sharkia \\
2
\end{tabular} & Menofiya & & & \\
\hline Sakha 93 & 22.93 & 17.44 & 15.14 & 20.93 & 23.73 & 13.57 & 21.67 & 27.40 & 15.20 & 19.78 \\
\hline Gemmeiza 9 & 24.2 & 17.94 & 14.87 & 21.27 & 24.73 & 14.1 & 22.34 & 21.03 & 20.64 & 20.12 \\
\hline Giza 168 & 25.29 & 17.77 & 13.14 & 23.2 & 24.03 & 14.07 & 26.67 & 17.33 & 20.83 & 20.26 \\
\hline Checks mean & 24.14 & 17.71 & 14.38 & 21.8 & 24.16 & 13.91 & 23.56 & 21.92 & 18.89 & 20.05 \\
\hline Gemmeiza 12 & 27.43 & 18.18 & 14.80 & 22.80 & 21.00 & 15.30 & 23.67 & 22.35 & 21.35 & 20.76 \\
\hline C.V \% & 5.93 & 6.1 & 9.78 & 4.71 & 6.41 & 9.86 & 13.23 & 9.39 & 2.48 & 7.54 \\
\hline L.S.D. at $5 \%$ & 2.2 & 1.59 & 1.93 & 1.57 & 2.15 & 2.1 & 4.45 & 3.53 & 0.65 & 2.24 \\
\hline
\end{tabular}

Table (3-c): Grain yield (ard / fed.) of the advanced yield trials (D-BW) for Gemmeiza 12 and three bread wheat cultivars at Middle Egypt in 2009/2010 season

\begin{tabular}{|l|c|c|c|c|c|c|}
\hline \multirow{2}{*}{ Cultivars } & \multicolumn{5}{|c|}{ Locations } & \multirow{2}{*}{ Mean } \\
\cline { 2 - 6 } & Giza & Fayoum & Sids & Mallawy & Menia & \\
\hline Sakha 93 & 26.8 & 14 & 24.01 & 17.42 & 18.94 & 20.23 \\
\hline Gemmeiza 9 & 26.2 & 19.33 & 22.63 & 18.32 & 18.67 & 21.03 \\
\hline Giza 168 & 26.85 & 19.33 & 19.35 & 18.14 & 19.07 & 20.55 \\
\hline Checks mean & 26.62 & 17.55 & 21.99 & 17.96 & 18.9 & 20.6 \\
\hline Gemmeiza 12 & $\mathbf{2 7 . 0 0}$ & $\mathbf{2 0 . 0 0}$ & $\mathbf{2 5 . 3 9}$ & $\mathbf{1 8 . 3 8}$ & $\mathbf{1 9 . 8 9}$ & $\mathbf{2 2 . 1 1}$ \\
\hline C.V\% & 4.25 & 14.73 & 8.76 & 7.09 & 6.95 & 8.36 \\
\hline L.S.D. at 5\% & 1.52 & 3.94 & 2.99 & 1.86 & 1.86 & 2.43 \\
\hline
\end{tabular}

In Out Valley zone (Table 3-f), Gemmeiza 12 proved its superiority over the check cultivars Sakha 93, Gemmeiza 9 and Giza 168 at Assuit, Ismailia and El-Ewinat locations. Meanwhile, its productivity was less than all check cultivars at Nubaria location and gave more yield than Sakha 93 cultivar at El-Bostan location as well as Giza 168 at New Valley and fewer yields than Gemmeiza 9 at both locations. The grain yield productivity was in favor of Gemmeiza 12 by $1.24 \mathrm{ard} / \mathrm{fed}(9.1 \%)$ at over all Out Valley regions. 
The new released cultivar outyielded the mean yield of all the check cultivars in 34 advanced yield trials at over all Egypt in 2009/2010 season by $4.1 \%$ (Table 3-j).

Table (3-d): Grain yield (ard/fed.) of the advanced yield trials(D-BW)for Gemmeiza 12and three bread wheat cultivars at Upper Egypt in 2009/2010 season.

\begin{tabular}{|l|c|c|c|c|}
\hline \multicolumn{1}{|c|}{ Cultivars } & \multicolumn{3}{|c|}{ Locations } & \multirow{2}{*}{ Mean } \\
\cline { 2 - 4 } & Shandaweel & Mattana & Kom-Ombo & \\
\hline Sakha 93 & 18.87 & 16.87 & 13.69 & 16.47 \\
\hline Gemmeiza 9 & 17.97 & 20 & 17.6 & 18.52 \\
\hline Giza 168 & 19.05 & 18.67 & 17.8 & 18.51 \\
\hline Checks mean & 18.63 & 18.51 & 16.36 & 17.83 \\
\hline Gemmeiza 12 & $\mathbf{1 7 . 6 3}$ & $\mathbf{1 9 . 0 0}$ & $\mathbf{1 5 . 2 2}$ & $\mathbf{1 7 . 2 8}$ \\
\hline C.V\% & 7.69 & 10.42 & 15.82 & 11.31 \\
\hline L.S.D. at 5\% & 2.14 & 2.79 & 3.59 & 2.84 \\
\hline
\end{tabular}

Table (3-e): Grain yield (ard / fed.) of the advanced yield trials (D-BWDrill )for Gemmeiza 12 and three bread wheat cultivars in 2009/2010 season.

\begin{tabular}{|l|c|c|c|c|c|c|}
\hline \multirow{2}{*}{ Cultivars } & \multicolumn{5}{|c|}{ locations } & \multirow{2}{*}{ Mean } \\
\cline { 2 - 6 } & Gemmeiza & Sakha & Giza & Sids & Shandaweel & \\
\hline Sakha 93 & 23.5 & 21.95 & 17 & 30.76 & 22.22 & 23.09 \\
\hline Gemmeiza 9 & 27.07 & 21.45 & 19.83 & 29.35 & 21.39 & 23.82 \\
\hline Giza 168 & 27.8 & 21.94 & 18.33 & 28.31 & 20.59 & 23.39 \\
\hline Checks mean & 26.12 & 21.78 & 18.39 & 29.47 & 21.4 & 23.43 \\
\hline Gemmeiza 12 & $\mathbf{2 9 . 7 0}$ & $\mathbf{2 2 . 3 3}$ & $\mathbf{1 8 . 3 3}$ & $\mathbf{3 0 . 6 9}$ & $\mathbf{2 1 . 7 7}$ & $\mathbf{2 4 . 5 6}$ \\
\hline C.V\% & 10.41 & 5.00 & 10.51 & 4.91 & 8.62 & 7.89 \\
\hline L.S.D. at 5\% & 4.10 & 1.60 & 2.83 & 2.11 & 2.84 & 2.70 \\
\hline
\end{tabular}

Table (3-f): Grain yield (ard / fed.) of the advanced yield trials (D-BW) for Gemmeiza 12 and three bread wheat cultivars at Out Valley in 2009/2010 season.

\begin{tabular}{|l|c|c|c|c|c|c|c|}
\hline Cultivars & \multicolumn{1}{l}{ locations } & \multirow{2}{*}{ Mean } \\
\cline { 2 - 7 } & Nubaria & $\begin{array}{l}\text { Al- } \\
\text { Bostan }\end{array}$ & Assuit & $\begin{array}{l}\text { New } \\
\text { valley }\end{array}$ & Ismalia & $\begin{array}{l}\text { El- } \\
\text { Ewinat }\end{array}$ & \\
\hline Sakha 93 & 20.75 & 10.00 & 7.20 & 21.18 & 6.92 & 15.77 & 13.64 \\
\hline Gemmeiza 9 & 21.27 & 12.47 & 6.00 & 20.35 & 8.39 & 16.01 & 14.08 \\
\hline Giza 168 & 22.93 & 11.40 & 6.40 & 12.69 & 8.00 & 16.96 & 13.06 \\
\hline Checks mean & 21.65 & 11.29 & 6.53 & 18.07 & 7.77 & 16.25 & 13.59 \\
\hline Gemmeiza 12 & $\mathbf{2 0 . 6 0}$ & $\mathbf{1 1 . 0 7}$ & $\mathbf{7 . 7 3}$ & $\mathbf{2 0 . 1 1}$ & $\mathbf{8 . 4 8}$ & $\mathbf{2 0 . 9 7}$ & $\mathbf{1 4 . 8 3}$ \\
\hline C.V\% & 11.04 & 12.12 & 6.30 & 3.31 & 6.34 & 4.18 & 7.21 \\
\hline L.S.D. at 5\% & 3.35 & 1.93 & 0.95 & 0.85 & 0.79 & 1.05 & 1.49 \\
\hline
\end{tabular}

The average grain yield of Gemmeiza 12 was $20.97 \mathrm{ard} / \mathrm{fed}$ over three growing seasons of 2007/2008, 2008/2009 and 2009/2010. The mean yield of the new released cultivar out yielded all the checks mean, Sakha 93,

$$
17.9
$$


Gemmeiza 9 and Giza 168. However, its yield productivity was insignificantly less than Sids 12 over two growing seasons of 2007/2008 and 2008/2009 (Table 4).

Table (3-j): Grain yield (ard / fed.) of the advanced yield trials (D-BW) for Gemmeiza 12 and three bread wheat cultivars at over all Egypt in 2009/2010 season.

\begin{tabular}{|l|c|c|c|c|c|c|c|}
\hline \multirow{2}{*}{ Cultivars } & $\begin{array}{c}\text { North } \\
\text { Delta }\end{array}$ & $\begin{array}{c}\text { South } \\
\text { Delta }\end{array}$ & $\begin{array}{c}\text { Middle } \\
\text { Egypt }\end{array}$ & $\begin{array}{c}\text { Upper } \\
\text { Egypt }\end{array}$ & $\begin{array}{c}\text { Out } \\
\text { valley }\end{array}$ & Drill & Mean \\
\hline Sakha 93 & 15.6 & 19.78 & 20.23 & 16.47 & 13.64 & 23.09 & 18.14 \\
\hline Gemmeiza 9 & 17.28 & 20.12 & 21.03 & 18.52 & 14.08 & 23.82 & 19.14 \\
\hline Giza 168 & 16.67 & 20.26 & 20.55 & 18.51 & 13.06 & 23.39 & 18.74 \\
\hline Checks mean & 16.52 & 20.05 & 20.6 & 17.83 & 13.59 & 23.43 & 18.67 \\
\hline Gemmeiza 12 & $\mathbf{1 7 . 0 1}$ & $\mathbf{2 0 . 7 6}$ & $\mathbf{2 2 . 1 1}$ & $\mathbf{1 7 . 2 8}$ & $\mathbf{1 4 . 8 3}$ & $\mathbf{2 4 . 5 6}$ & $\mathbf{1 9 . 4 3}$ \\
\hline C.V \% & 11.61 & 7.54 & 8.36 & 11.31 & 6.71 & 7.89 & 8.90 \\
\hline L.S.D. at 5\% & 2.47 & 2.24 & 2.43 & 2.84 & 1.33 & 2.70 & 2.34 \\
\hline
\end{tabular}

Table 4: Average grain yield (ard/fed) for Gemmeiza 12 and four bread wheat cultivars over three growing seasons.

\begin{tabular}{|l|c|c|c|c|}
\hline \multirow{2}{*}{ Cultivars } & \multicolumn{3}{|c|}{ years } & \multirow{2}{*}{ Mean } \\
\cline { 2 - 4 } & $\mathbf{2 0 0 7 / 2 0 0 8}$ & $\mathbf{2 0 0 8 / 2 0 0 9}$ & $\mathbf{2 0 0 9 / 2 0 1 0}$ & \\
\hline Sakha 93 & 19.16 & 21.73 & 18.14 & 19.68 \\
\hline Gemmeiza 9 & 19.81 & 22.18 & 19.14 & 20.38 \\
\hline Giza 168 & 19.38 & 22.21 & 18.74 & 20.11 \\
\hline Sids 12 & 20.65 & 22.79 & - & 21.72 \\
\hline Checks mean & 19.75 & 22.23 & 18.67 & 20.22 \\
\hline Gemmeiza 12 & $\mathbf{2 0 . 6 3}$ & $\mathbf{2 2 . 8 4}$ & $\mathbf{1 9 . 4 3}$ & $\mathbf{2 0 . 9 7}$ \\
\hline C.V\% & 11.82 & 8.05 & 8.90 & 9.59 \\
\hline L.S.D. at 5\% & 2.28 & 2.45 & 2.34 & 2.35 \\
\hline
\end{tabular}

\section{3-Verification yield trials:}

The results presented in table 5-a show the average grain yield for Gemmeiza 12 and four commercial wheat cultivars in 23 on-farm yield trials grown in different governorates represent all production areas in Egypt in 2010/2011 growing season. The obtained data confirm the results we got in yield trials which support the superiority of Gemmeiza 12 over the dominating wheat cultivars at Delta region and compete with Sids 12, Giza 168 and Sakha 93 at Upper Egypt and yielded the same as Sakha 93 at New lands .

The results of 32 on - farm trials in 2011/2012 which are presented in Table 5-b showed that Gemmeiza 12 surpassed the check cultivars at Delta region while its productivity was less than Gemmeiza 9 at Upper Egypt and new lands. In 2012/2013 growing season, the grain yield of Gemmeiza 12 overcome Giza 168 and Sids 12 at Delta region as well as Giza 168 in the new lands(Table 5-c). 
Table (5-a): Grain yield (ard / fed.) of verification yield trials of the newly released cultivar Gemmeiza 12 in 2010 /2011 season.

\begin{tabular}{|c|c|c|c|c|c|c|c|}
\hline Zones & Governorates & $\begin{array}{c}\text { No. of } \\
\text { trials }\end{array}$ & Sakha 93 & Gemmeiza 9 & Giza 168 & Sids12 & Gemmeiza12 \\
\hline \multirow[b]{2}{*}{ North Delta } & El-Behera & 2 & 20.02 & 19.95 & 21.70 & 22.12 & 22.12 \\
\hline & $\begin{array}{l}\text { Kafr } \\
\text { El -Sheikh }\end{array}$ & 2 & 17.80 & 18.20 & 19.28 & - & - \\
\hline \multicolumn{3}{|l|}{ Mean } & 18.91 & 19.08 & 20.49 & 22.12 & 22.12 \\
\hline \multirow{3}{*}{ Middle Delta } & Sharkia & 2 & 16.05 & 14.00 & 17.76 & 22.15 & 21.52 \\
\hline & Dakahlia & 2 & 18.31 & 20.98 & 14.20 & 19.91 & 19.35 \\
\hline & Gharbia & 2 & 22.80 & 22.40 & 23.40 & 25.2 & 28.00 \\
\hline \multicolumn{3}{|l|}{ Mean } & 19.05 & 19.13 & 18.45 & 22.42 & 22.96 \\
\hline \multirow{3}{*}{ South Delta } & Monufia & 2 & 23.32 & 23.24 & 22.26 & 22.49 & 23.50 \\
\hline & Qalyubia & 2 & 24.11 & 24.91 & 26.57 & 26.60 & 25.58 \\
\hline & Giza & 2 & 25.20 & 25.90 & 25.90 & 23.80 & 24.30 \\
\hline \multicolumn{3}{|l|}{ Mean } & 24.21 & 24.68 & 24.91 & 24.30 & 25.06 \\
\hline \multirow{3}{*}{ Upper Egypt } & El-Menia & 2 & 23.20 & 23.19 & 19.76 & 27.16 & 25.34 \\
\hline & Sohag & 2 & 17.92 & - & 20.16 & 15.68 & 18.02 \\
\hline & Aswan & 1 & 16.80 & - & 16.00 & 16.00 & 14.00 \\
\hline \multicolumn{3}{|l|}{ Mean } & 19.31 & 23.91 & 18.64 & 19.61 & 19.12 \\
\hline \multirow{2}{*}{ New Lands } & Port Said & 1 & 18.44 & 24.35 & 17.81 & 20.27 & 16.47 \\
\hline & Nubaria & 1 & 13.89 & 19.25 & 15.58 & 19.39 & 17.36 \\
\hline \multicolumn{3}{|l|}{ Mean } & 16.17 & 21.80 & 15.69 & 16.86 & 16.92 \\
\hline \multicolumn{3}{|l|}{ Zones mean } & 19.53 & 21.72 & 19.84 & 21.66 & 21.24 \\
\hline
\end{tabular}

Table (5-b): Grain yield (ard / fed.) of verification yield trials of the newly released cultivar Gemmeiza 12 in 2011 /2012 season.

\begin{tabular}{|c|c|c|c|c|c|c|}
\hline Zones & Governorates & $\begin{array}{l}\text { No. of } \\
\text { trials }\end{array}$ & Gemmeiza9 & Giza 168 & Sids12 & Gemmeiza12 \\
\hline \multirow{3}{*}{ North Delta } & El-Beheira & 2 & 21.28 & 20.86 & 24.50 & 24.22 \\
\hline & Alexandria & 2 & 22.68 & 22.26 & 20.86 & 21.39 \\
\hline & \begin{tabular}{|l|} 
Kafr \\
El -Sheikh
\end{tabular} & 4 & 19.33 & 22.38 & 18.12 & 20.50 \\
\hline \multicolumn{3}{|l|}{ Mean } & 21.10 & 21.83 & 21.16 & 22.04 \\
\hline \multirow{2}{*}{ Middle Delta } & Dakahlia & 2 & 23.80 & 21.75 & 23.76 & 20.75 \\
\hline & Gharbia & 2 & 30.00 & 22.23 & 25.36 & 25.72 \\
\hline \multicolumn{3}{|l|}{ Mean } & 26.9 & 21.99 & 24.56 & 23.24 \\
\hline \multirow{3}{*}{ South Delta } & Monufia & 3 & 20.81 & 24.12 & 25.25 & 26.35 \\
\hline & Qalyubia & 2 & 28.51 & 24.98 & 27.72 & 30.20 \\
\hline & Giza & 1 & 27.30 & 29.05 & 29.40 & 27.30 \\
\hline \multicolumn{3}{|l|}{ Mean } & 25.54 & 26.05 & 27.46 & 27.95 \\
\hline \multirow{2}{*}{ Middle Egypt } & Faiyum & 2 & - & 27.65 & 29.30 & 26.10 \\
\hline & Beni-suef & 2 & - & 19.25 & 18.65 & 20.55 \\
\hline \multicolumn{3}{|l|}{ Mean } & - & 23.45 & 23.98 & 23.33 \\
\hline \multirow{4}{*}{ Upper Egypt } & El-Menia & 2 & - & 21.41 & 22.10 & 22.10 \\
\hline & Assiut & 2 & - & 24.36 & 19.57 & 22.70 \\
\hline & Sohag & 2 & - & 22.40 & 23.24 & 19.60 \\
\hline & Aswan & 1 & - & 16.80 & 18.30 & 13.70 \\
\hline \multicolumn{3}{|l|}{ Mean } & - & 21.24 & 20.80 & 19.53 \\
\hline \multirow{4}{*}{ New Lands } & Ismailia & 1 & 24.50 & 21.00 & 24.50 & 21.70 \\
\hline & Port Said & 1 & 22.93 & 17.86 & 24.38 & 19.18 \\
\hline & Suez & 1 & 17.92 & 17.64 & 18.70 & 18.48 \\
\hline & Nubaria & 1 & 14.93 & 17.73 & 20.53 & 20.53 \\
\hline \multicolumn{3}{|l|}{ Mean } & 19.12 & 18.79 & 21.24 & 20.34 \\
\hline \multicolumn{3}{|l|}{ Zones mean } & 23.17 & 22.23 & 23.20 & 22.80 \\
\hline
\end{tabular}


Table (5-c): Grain yield (ard./ fed.) of verification yield trials of the newly released cultivar Gemmeiza 12 in 2012 /2013 season.

\begin{tabular}{|c|c|c|c|c|c|}
\hline Zones & Governorates & $\begin{array}{l}\text { No. of } \\
\text { trials }\end{array}$ & Giza 168 & Sids12 & Gemmeiza12 \\
\hline \multirow{4}{*}{ North Delta } & El-Behera & 1 & 21.21 & 22.40 & 20.72 \\
\hline & $\begin{array}{l}\text { Kafr } \\
\text { EI -Sheikh }\end{array}$ & 2 & 18.83 & 17.99 & 17.90 \\
\hline & Alexandria & 1 & 18.55 & 15.89 & 23.73 \\
\hline & Damietta & 1 & 19.83 & 17.90 & 19.25 \\
\hline \multicolumn{3}{|l|}{ Mean } & 19.60 & 18.54 & 20.4 \\
\hline \multirow{3}{*}{ Middle Delta } & Sharkia & 2 & 22.47 & 22.94 & 19.74 \\
\hline & Gharbia & 1 & 22.40 & 22.40 & 23.80 \\
\hline & Dakhalia & 2 & 19.33 & 19.13 & 26.59 \\
\hline \multicolumn{3}{|l|}{ Mean } & 21.40 & 21.49 & 23.38 \\
\hline \multirow{2}{*}{ South Delta } & Monufia & 2 & 22.26 & 22.56 & 23.88 \\
\hline & Qalyubia & 2 & 24.72 & 30.42 & 30.21 \\
\hline \multicolumn{3}{|l|}{ Mean } & 23.49 & 26.49 & 27.04 \\
\hline \multirow{4}{*}{ New Lands } & Port Said & 1 & 16.67 & 23.05 & 21.94 \\
\hline & Suez & 1 & 19.60 & 20.44 & 18.48 \\
\hline & Alexandria & 1 & 22.82 & 19.40 & 18.97 \\
\hline & \begin{tabular}{|l} 
Kafr \\
El -Sheikh
\end{tabular} & 1 & 17.22 & 22.68 & 17.08 \\
\hline \multicolumn{3}{|l|}{ Mean } & 19.07 & 21.39 & 19.11 \\
\hline \multicolumn{3}{|l|}{ Zones mean } & 20.89 & 21.98 & 22.48 \\
\hline
\end{tabular}

\section{4-Demonstration fields:}

It is clear from the results of 22 demonstration fields presented in Table 6 that the new cultivar Gemmeiza 12 yielded $25.70 \mathrm{ard} / \mathrm{fed}$ and surpassed the yield of the check cultivars Gemmeiza 9, Giza 168 and Sids 12 in the demonstration fields at four governorates representing Delta region i.e. , Gharbia, Monufia, Dakhalia and Qalyubia. The grain yield of Gemmeiza 12 exceeded the mean yield of the four governorates.

Table (6): Average grain yield (ard/fed) of Gemmeiza 12 and four commerical wheat cultivars at four governorates in 2012/2013 season.

\begin{tabular}{|l|c|c|c|}
\hline Cultivars & $\begin{array}{c}\text { Demonstration field } \\
\text { (ard/fed) }\end{array}$ & Governorate & $\begin{array}{c}\text { Mean * } \\
\text { (ard/fed) }\end{array}$ \\
\hline Gemmeiza 9 & 24.24 & Gharbia & 20.77 \\
\hline Giza 168 & 22.79 & Monufia & 22.10 \\
\hline Sids 12 & 24.46 & Dakhalia & 19.37 \\
\hline Gemmeiza 12 & 25.70 & Qalyubia & 19.10 \\
\hline
\end{tabular}

${ }^{*}$ Central Administration of Agricultural Statistic (CAAS)

\section{5-Rust disease resistance:}

Tables 7-a, b and c showed disease rust resistance scores for the new released wheat cultivar Gemmeiza 12 at Gemmeiza, Sakha and Nubaria Agric. Res. Stations during 2007/2008, 2008/2009 and 2009/2010 growing seasons. Performance of Gemmeiza 12 reflecting good resistance for both 
leaf and stem rust diseases comparing with the dominating wheat cultivars. Table 7-d reveal the mean average coefficient of leaf and stem rust diseases of Gemmeiza 12 under field conditions at three stations. The data showed that the new cultivar Gemmeiza 12 exhibited the lowest values of mean $\mathrm{ACl}$ for leaf and stem rust diseases being 0.66 and 0.00 respectively compared with the check cultivars Sakha 93, Gemmeiza 9 and Giza 168.

Table (7-a): leaf and stem rust disease severity at adult stage for Gemmeiza 12 and four bread wheat cultivars at Gemmeiza, Sakha and Nubaria Res. Stations in 2007/008 season.

\begin{tabular}{|l|c|c|c|c|c|c|}
\hline Cultivar & \multicolumn{2}{|c|}{ Gemmeiza } & \multicolumn{2}{c|}{ Sakha } & \multicolumn{2}{c|}{ Nubaria } \\
\cline { 2 - 7 } & $\begin{array}{c}\text { Leaf } \\
\text { rust }\end{array}$ & $\begin{array}{c}\text { Stem } \\
\text { rust }\end{array}$ & $\begin{array}{c}\text { Leaf } \\
\text { rust }\end{array}$ & $\begin{array}{c}\text { Stem } \\
\text { rust }\end{array}$ & $\begin{array}{c}\text { Leaf } \\
\text { rust }\end{array}$ & $\begin{array}{c}\text { Stem } \\
\text { rust }\end{array}$ \\
\hline Sakha 93 & $80 \mathrm{~S}$ & 0 & $5 \mathrm{~S}$ & 0 & $10 \mathrm{MR}$ & 0 \\
\hline Gemmeiza 9 & 0 & 0 & $5 \mathrm{R}$ & 0 & $10 \mathrm{MR}$ & 0 \\
\hline Giza 168 & 0 & 0 & Tr.R & 0 & 0 & 0 \\
\hline Sids 12 & 0 & 0 & Tr.MS & 0 & Tr.R & 0 \\
\hline Gemmeiza 12 & 0 & 0 & Tr.R & 0 & Tr.R & 0 \\
\hline
\end{tabular}

Table (7-b): leaf and stem rust disease severity at adult stage for Gemmeiza 12 and four bread wheat cultivars at Gemmeiza, Sakha and Nubaria Res. Stations in 2008/009 season.

\begin{tabular}{|l|c|c|c|c|c|c|}
\hline Cultivar & \multicolumn{2}{|c|}{ Gemmeiza } & \multicolumn{2}{c|}{ Sakha } & \multicolumn{2}{c|}{ Nubaria } \\
\cline { 2 - 7 } & $\begin{array}{c}\text { Leaf } \\
\text { rust }\end{array}$ & $\begin{array}{c}\text { Stem } \\
\text { rust }\end{array}$ & $\begin{array}{c}\text { Leaf } \\
\text { rust }\end{array}$ & $\begin{array}{c}\text { Stem } \\
\text { rust }\end{array}$ & $\begin{array}{c}\text { Leaf } \\
\text { rust }\end{array}$ & $\begin{array}{c}\text { Stem } \\
\text { rust }\end{array}$ \\
\hline Sakha 93 & $80 \mathrm{~S}$ & 0 & $30 \mathrm{~S}$ & 0 & $10 \mathrm{MR}$ & 0 \\
\hline Gemmeiza 9 & $30 \mathrm{~S}$ & 0 & $5 \mathrm{MS} / \mathrm{S}$ & 0 & $5 \mathrm{MR} / \mathrm{MS}$ & 0 \\
\hline Giza 168 & 0 & $10 \mathrm{MR}$ & 0 & $5 \mathrm{MS}$ & $\mathrm{Tr} . \mathrm{R}$ & $5 \mathrm{MS}$ \\
\hline Sids 12 & $10 \mathrm{~S}$ & $40 \mathrm{~S}$ & $10 \mathrm{MR} / \mathrm{MS}$ & $10 \mathrm{~S}$ & $10 \mathrm{MR}$ & 0 \\
\hline Gemmeiza 12 & 0 & 0 & $\mathrm{Tr} . \mathrm{MR}$ & 0 & $10 \mathrm{MS} / \mathrm{S}$ & $5 \mathrm{MR} / \mathrm{MS}$ \\
\hline
\end{tabular}

Table (7-c): leaf and stem rust disease severity at adult stage for Gemmeiza 12 and four bread wheat cultivars at Gemmeiza, Sakha and Nubaria Res. Stations in 2009/2010 season.

\begin{tabular}{|l|c|c|c|c|c|c|}
\hline \multirow{2}{*}{ Cultivar } & \multicolumn{2}{|c|}{ Gemmeiza } & \multicolumn{2}{c|}{ Sakha } & \multicolumn{2}{c|}{ Nubaria } \\
\cline { 2 - 7 } & $\begin{array}{c}\text { Leaf } \\
\text { rust }\end{array}$ & $\begin{array}{c}\text { Stem } \\
\text { rust }\end{array}$ & $\begin{array}{c}\text { Leaf } \\
\text { rust }\end{array}$ & $\begin{array}{c}\text { Stem } \\
\text { rust }\end{array}$ & $\begin{array}{c}\text { Leaf } \\
\text { rust }\end{array}$ & $\begin{array}{c}\text { Stem } \\
\text { rust }\end{array}$ \\
\hline Sakha 93 & $30 \mathrm{~S}$ & 0 & $30 \mathrm{~S}$ & 0 & $5 \mathrm{MS}$ & TR.MR \\
\hline Gemmeiza 9 & $10 \mathrm{MS}$ & 0 & $20 \mathrm{~S}$ & 0 & $5 \mathrm{MS}$ & TR.S \\
\hline Giza 168 & 0 & TR.MS & Tr.S & 0 & TR.MR & TR.MS \\
\hline Sids 12 & 0 & 0 & 0 & 0 & 0 & 0 \\
\hline Gemmeiza 12 & $10 \mathrm{R}$ & 0 & 0 & 0 & 0 & 0 \\
\hline
\end{tabular}


Table (7-d): Mean average coefficient of leaf and stem rust disease infection ( $\mathrm{ACl})$ at adult stage for Gemmeiza 12 and four bread wheat cultivars at Gemmeiza, Sakha and Nubaria Res. Stations in 2009/2010 season.

\begin{tabular}{|l|c|c|c|c|c|c|c|c|}
\hline Cultivar & \multicolumn{2}{|c|}{ Gemmeiza } & \multicolumn{2}{c|}{ Sakha } & \multicolumn{2}{c|}{ Nubaria } & \multicolumn{2}{c|}{ Mean ACI } \\
\cline { 2 - 9 } & $\begin{array}{c}\text { Leaf } \\
\text { rust }\end{array}$ & $\begin{array}{c}\text { Stem } \\
\text { rust }\end{array}$ & $\begin{array}{c}\text { Leaf } \\
\text { rust }\end{array}$ & $\begin{array}{c}\text { Stem } \\
\text { rust }\end{array}$ & $\begin{array}{c}\text { Leaf } \\
\text { rust }\end{array}$ & $\begin{array}{c}\text { Stem } \\
\text { rust }\end{array}$ & $\begin{array}{c}\text { Leaf } \\
\text { rust }\end{array}$ & $\begin{array}{c}\text { Stem } \\
\text { rust }\end{array}$ \\
\hline Sakha 93 & 30.00 & 0.00 & 30.00 & 0.00 & 4.00 & 0.80 & 21.33 & 0.26 \\
\hline Gemmeiza 9 & 8.00 & 0.00 & 20.00 & 0.00 & 4.00 & 2.00 & 10.66 & 0.66 \\
\hline Giza 168 & 0.00 & 1.60 & 2.00 & 0.00 & 0.80 & 1.60 & 0.93 & 1.06 \\
\hline Sids 12 & 0.00 & 0.00 & 0.00 & 0.00 & 0.00 & 0.00 & 0.00 & 0.00 \\
\hline Gemmeiza 12 & $\mathbf{2 . 0 0}$ & $\mathbf{0 . 0 0}$ & $\mathbf{0 . 0 0}$ & $\mathbf{0 . 0 0}$ & $\mathbf{0 . 0 0}$ & $\mathbf{0 . 0 0}$ & $\mathbf{0 . 6 6}$ & $\mathbf{0 . 0 0}$ \\
\hline
\end{tabular}

\section{6-Technological and quality characters:}

Technological and quality characters for the new wheat cultivar Gemmeiza 12 were carried out by Field Crops Technology Research Department, Food Technology Research Institute, ARC. The results showed that milling performance for Gemmeiza 12 has been good, since it had the highest hectoliter value being $83.9 \mathrm{~kg} / \mathrm{hl}$. with flour yield often higher than all check cultivars i.e, Sakha 93, Gemmeiza 9, Giza 168 and Sids 12 .Protein content of the grain has been $1 \%$ higher than Giza 168 and about comparable with Gemmeiza 9 .The flour of Gemmeiza 12 is acceptable for bread making, since the protein content reached about $13 \%$ and the wet gluten percentage was $29.1 \%$ (Table 8 ).

Table (8): Some technological and quality characters of the new bread wheat Gemmeiza 12 and four commercial wheat cultivars:

\begin{tabular}{|l|c|c|c|c|c|c|}
\hline Cultivar & $\begin{array}{c}\text { Hectoliter } \\
\text { weight } \\
\text { Kg/hl }\end{array}$ & Protein\% & Ash\% & $\begin{array}{c}\text { Extraction } \\
\text { Rate\% }\end{array}$ & Wet & Dry \\
\hline Sakha93 & 82.1 & 10.0 & 1.8 & 70.0 & 21.8 & 7.2 \\
\hline Gemmieza9 & 81.0 & 12.6 & 1.7 & 68.5 & 30.8 & 10.3 \\
\hline Giza168 & 82.1 & 12.0 & 1.8 & 68.3 & 28.8 & 9.1 \\
\hline Sids 12 & 82.1 & 10.8 & 1.8 & 70.0 & 23.2 & 8.0 \\
\hline Checks mean & 82.3 & 11.7 & 1.7 & 69.4 & 26.9 & 8.96 \\
\hline Gemmeiza 12 & 83.9 & 13.0 & 1.6 & 71.0 & 29.1 & 9.8 \\
\hline
\end{tabular}

\section{7-Description of Gemmeiza 12:}

Gemmeiza 12 has a spring growth habit with medium maturity. Tillering is profuse and leaves are moderately narrow. The spikes are fully awns and mid dense. The variety is somewhat medium tall. Kernels color is white, medium in size and tends to have a hard texture. The morphological characteristics of the new released cultivar shown in table 9 were carried out by the Central Administration of Seed Certification (CASC) according to the International Union for the Protection of new Varieties of plants (UPOV). The Distinctness Uniformity and Stability (DUS) test should be done before releasing the variety. 
Table 9: Some morphological characteristics of the new released wheat cultivar Gemmeiza 12 according to the International Union for the Protection of new Varieties of plants (UPOV)

\begin{tabular}{|c|c|c|}
\hline No. & Characteristics & Description \\
\hline 1 & Pigmentation of coleoptile & Very weak (1) \\
\hline 2 & Anthocyanin coloration of auricles & Very weak (1) \\
\hline 3 & Plant growth habit & Erect (1) \\
\hline 4 & Flag leaf rolling & Medium (5) \\
\hline 5 & Time of ear emergence (first spike on $50 \%$ of ears) & Early (5) \\
\hline 6 & Glaucosity of flag leaf sheath & Strong (7) \\
\hline 7 & Glaucosity of flag leaf blade & Medium (5) \\
\hline 8 & Ear: anthocyanin pigmentation & Very weak (1) \\
\hline 10 & Glaucosity of ear neck & Strong (7) \\
\hline 11 & Glaucosity of ear & Medium (5) \\
\hline 12 & Plant height (stem, ear and awns) & Medium (5) \\
\hline 13 & Awns presence & Present (3) \\
\hline 14 & Awns length & Medium (5) \\
\hline 15 & Lower glume: Shoulder shape & Sloping (1) \\
\hline 16 & Lower glume: Shoulder width & Narrow (3) \\
\hline 17 & Lower glume: beak length & Short (3) \\
\hline 18 & Lower glume: beak shape & geniculate (5) \\
\hline 19 & Lower glume: extent of internal hairs & Week(3) \\
\hline 20 & Lowest lemma: beak shape & Slightly curved(3) \\
\hline 21 & Straw : pith in cross section & Thin(3) \\
\hline 22 & Ear length (ear and awns) & Medium (5) \\
\hline 23 & Ear: hair density at the lower edge of the rachis & Weak (3) \\
\hline 24 & Shape of ear & Tapering (1) \\
\hline 25 & Density of ear & Dense (7) \\
\hline 26 & Grain color & White (1) \\
\hline 27 & Length of grain brush hairs & Short (3) \\
\hline 28 & Seasonal type & Spring type (3) \\
\hline
\end{tabular}

In conclusion, we can strongly recommend the new bread wheat cultivar Gemmeiza 12 to be grown at North Delta, South Delta and Middle Egypt, since its productivity overcome most of the dominating wheat cultivars i.e, Sakha 93, Gemmeiza 9, Giza 168 and Sids 12. In addition, the new cultivar surpassed the dominating wheat cultivar Sakha 93 at all locations representing Upper Egypt and Out Valley zones however, its productivity was slightly less than Giza 168 and Sids 12 in both locations. The performance of Gemmeiza 12 was good when using drill planting at Sakha, Gemmeiza, Giza, Sids and Shandaweel Agriculture Research Stations. The relative grain yield of four commercial wheat cultivars in comparison with Gemmeiza 12 in 76 yield trials over three years period proved that grain yield of Gemmeiza 12 was $3.7 \%$ higher than the commercial wheat cultivar means in the major wheat production areas of Egypt.

Seeds of Gemmeiza 12 will be available for planting in 2014/2015 season. Foundation seed will be maintained and distributed by Wheat Research Department, Field Crops Research Institute, ARC. Egypt. 


\section{REFERENCES}

Ageez, A.A.; Iman M.M. Sadek; M.S. Saleh and R.M. Mitkees.(1999).Genetic improvement in Egyptian wheat yield since 1950. J. Agric. Sci., Mansoura Univ., 24 (7) 3265-3278.

Comstock, R.E., and R.H.Moll.(1963).Genotype- environment interactions.PP.164-196.In:Hanson,W.D.and Robinson H.F.(ed) Statistical genetics and plant breeding. Nat. Acad. Sci., Nat. Res. Council. Washington, Dc.

El-Sayed, F.F.; Ali, A.M.A.S.; El.Menoufi, M.M.; Mitkees, R.A.;Hamada, A.A.; Mahrous, M.A.; Shehab El Din, T.M.; El Shami, M.M.; Mostafa, M.A.; Abdel- Ghani, A.M.; Eman M.M. Sadek;Ali, A.M.; Iskandar, M.H.; Bassiouni, A.H.; Eissa, A.M.K.; Abdel-Gawad, Y.G.; Kadria Hegazi and Y.H. El-Doudi, (1996): An Introduction to the new bread wheat cultivar Gemmeiza 3. J. Agric. Sci., Mansoura Univ.; 21 (11) 3811-3823.

El Shami, M.; T. Shehab El Din; M. Mostafa; M. Abdel Aleem,; M .Mahrous ; A. Ageez; A.Hamada; A. Bassiouni; M. Eid; A. Abdel- Ghani; M. Eskandar; S.Sabry; M. Sharshar; Iman Sadek; A. Abo- Warda; A.M. Mousa; E. El-Sayed ; H. Ashoush; M. Towfeeles; Hayam Mahjoub; A. Moustafa; H. Hendawy; F. Hefnawy; S. Ali; A. Abdel-Karim; A. Menshawy; H. El-Borhamy; M. Abdel- Fattah; G.A. El- Shaaray; S. ElSawi; R. Kumber; Sabah. Abo Alela; Waffa. El- Awady; I. Amin; A.Moussa; S. Abdel Dayem; M. Zakreia; S. Hammad; A. Swaliem; A. Gomaa; O. Khalil; Kadria Hegazi; Enayat Khanem; R. Mitkees; M. ElMenofy; A.M. Mousa; A. Abdel- Latif; N.Hanaa; A.Khattab and M. El Shami. (2005): Gemmeiza 10: A new Egyptian high yielding and rust resistant bread wheat cultivar, J.Agric. Sci., Mansoura Univ., 30:743754. 3265-3278.

Gomaa, A.S.; Khalil,O.S.; Abo Elenein. R.A.; Kadria F, Hegazi; Enayat H, Ghanem; Shafi Al, A.; El- Sayed, F.F.; Gouda, M.A. El Shami, M.; Ageez, A.A.; Saleh, M.E.; El- Hadidi, M.; Attia, S. and M.G.Mosaad (1984). Sakha 69 and Sakha 61: two new bread wheat high- yielding varieties. Second General Conference of ARC, Giza, Egypt, April 9 -11.

Mahrous, A.M.; M.Abdel- Aleem; T. Shehab El- Din; M. Mostafa;S. AbdelMajeed; S.Sabry; Iman M. Sadek; M.Sharshar; A. Hamada; A. AboWarda;A.M. Moussa; A. Tammam; M.Meshref; E.El-Sayed; H. Ashoush; M. Toweefles; H. Hendawy; Hayam S. Mahgoub; A. Mostafa; H. El-Borhamy;A.Menshawy; A.Moussa; Wafaa M. El-Awady; A.ElHag; R.Koumbor; S.Seleem; R.A.Ramadan; Nadia A.Abdel- Nour; G. Sharawy; Sohair M.Hassan; A. Sewelam; S. El- Saway; S. AbdelDayem; A. El-Sebaey; Magda A. Abdel-Rahman; Sabah. Abo Alela;M.Khaled; I.Amin; M. Zakaria; Manal A. Hassan; A. Gad-Allah; S. Hammada;M.El-Maghraby; A. Morad; Aza, M.Abdel -All; A. Hagras; A.T. Moustafa; M.Mahoud; M.Moubarak; Kadria Hegazi; A.Gomaa; O.Khalii; Enayat Khanem; R. Meetiks; M. El- Monofy; S. Kh. Mahmoud; N.Hanna; M.Eid; M.Mosaad;M.A.Gouds; A.Ageez; M.Abdel-Fattah; A.Khattab; A. A.Abdel-Lattif; M.Eskander; Najwa Abdel- Fattah; F. 
Hefnawy and W. Abdel- Samad.(2009).Grain yield and stability of the new Egyptian bread wheat cultivar Sids 12. J.Agric. Sci., Mansoura Univ., 34(4): 3199-3209.

Mitkees, R.A.; M.A. El-Menofy; A.A. Hamada.; F.F. El- Sayed.; A.A. Ageez; M.A. Mahrous; H. Ashoush; A.A Gomaa.; O.H.S Khalil.; Kadria F, Hegazi; Enayat H, Ghanem; A.A. Ali; M.G. Mosaad; M.M. El-Shami; A.H. Bassiouni; A.M.K. Eissa; T.M. Shehab El Din; M.M. Abde-Aleem; S.Kh. Mahmoud; M.A.M Eid; M.A. Mostafa; M.H. Iskandar; N.S. Hanna; S.R.S. Sabry; A.H. Abdel- Latif; A.M.A. Abo- Warda; Y.G. AbdelGwad; A.M. Mousa; S.A. Abdel- Majeed; H.M. Zaid; A.M. Tammam; Nagwa R. Abdel- Fatah; M.Kh. Mosherf; E.A.M. El-Sayed; Hayam S. Mahgoub; M.B. Toweefles; S. Abdel- Halim; A.K. Mostafa; F.A. Hefnawy; Y.H. El-Daoudi; M.O. Khilifa and M.M. El- Shamy(1998). Gemmeiza 5: A new Egyptian bread wheat cultivar. Annals of Agric. Sci.,Moshtohor, 36 :( 1) 43-59..

Mosaad M.; M. El- Menofy; T. Shehab El- Din; R.Mitkees; M. Mahrous; A. Hamada; A.Ageez; A. Bassiouni; M. El-Shami; M.Abdel-Aleem;M. Eid; A. Abdel- Ghani; M.Eskander; N. Hanaa;S.Sabry; A. Abdel-Latif; M.Sharshr; Iman Sadek; M.Mostafa;A.Abo-Warda; Y.Abdel-Gwad; A.Mousa; S.Abdel-Majeed;A.Tammam;NajwaAbdelFattah;M.Moshref;E.El-Sayed; H.Ashoush; M.Towfeeles; Hayam Mahjoub; A.Moustafa; F.Hefnawy; H. Hendawy; S.Ali; A.Abdel-Karim; A. Khattab; M.Abdel-Fattah; A. Menshawy; H.El-Borhamy; A.Gomaa;F. O. Khalil; Kadria Hegazi; A.Ali; Enayat Ghanem; S.Mahmoud and M.Khalifa(2000). Gemmeiza 9: A new Egyptian high yielding and rust resistant bread wheat cultivar for Delta region. J.Agric. Sci.,Mansoura Univ., 25 (12): 7407-7419.

Moustafa, M.A.; M.S.Sharshr; T. Shehab El- Din; M.Abo- Shereef; S.AbdelMajeed; M.Abdel -Aleem; S.R.S.Sabry; Iman M. Sadek; A. Hamada; A.Abo-Warda; A.Tammam; M.Moshref; E. El-Sayed; H.Ashoush; M.Towfeeles; H. Hendawy; Hayam S. Mahjoub; A.K. Moustafa; H.ElBorhamy; A. Menshawy; A.Moussa; Wafaa M. El-Awady; A. El-Hag; R.Koumbor; S. Seleem; R.A. Ramadan; Nadia A. Abdel- Nour; G.Sharawy; S. Abdel- Dayem; Sohair M. Hassan; A. Sewelam; S.ElSawy; S. Hammad; Magda A. Abdel- Rahman; S. Abo- Elela; M.A. Khaled; I.A. Amin; M. Zakaria; Manal A. Hassan; A. Gad- Allah; M.A. El- Maghraby; A.Morad; Aza M. Abdel- Al; A. Hagras; A. T. Moustafa; M. S. Mahmoud; M. Y. Mubark; Hoda M. M El- Gharbawy; A.A Mahmoud; A. Gomaa; Enayat Ghanem; R. Mitkees; M.El- Menofy; S.Kh. Mahmoud; N. Hanna; M.A. Moussa; M.A. Gouda; A. Ageez;M. A. Salem; A. Khattab; A. Abdel-Latif; M. Eskander; Najwa Abdel-Fattah; F. Hefnawy and W. Abdel-Samad(2010).Sids 13: A new bread wheat cultivar. J. of Plant Production,Mansoura Univ., 1 (1): 147-157. 
Saari, E. E. and R. D. Wilcoxson (1974). Plant disease situation of high yielding durum wheat in Asia and Africa. Arr. Rev. Phyto., 2:47-68.

Sadek ,Eman M.; A.Ageez; M.El-Menofy; M. Abo Shereef; A. Hamada; A.Moussa; R. Kumber; S. Sleem; G. El- Shaaray; A. A. morad; S. Abdel- Majeed; A. Abo- Warda; A.Tammam; M. Mesherf; E.A.M.ElSayed; H. Ashoush; M. Toweefles; H. Hendawy;Hayam S. Mahgoub; A.K. Mostafa; H. El-Borhamy; A. Menshawy; Wafaa M. El- Awady; Nadia Abd El-Nour; S. Abdel- Dayem; Sohair M. Hassan; A. Swelam; S. El- Sawy; S. Hamad;Magda A. Abdel - Rahman; Sabah. Abo El-Ela; M.A. Khaled; R.A. Ramadan; I. A. Amin; M. Zakaria; Manal A. Hassan; A. Gad-Allah; M. A. El-Maghraby; Aza M. Abdel-Al; A. Hagras; A.T. Mostafa; M.S. Mahmoud; M.Y.Moubark; Thanaa Abd-El-Kreem; A.M.Morsy; Hoda El-Gharabawy; AglanM.A.A; W. Farahat; Abd ElHamid E.A.M;Ragab K.I.Gad;E; Nathan Shereen;Abdel- Latif, I.S.M; Abdel Kader,M.N; A. Gomaa ;Enayat Ghanem; S.Kh. Mahmoud; M.G.Mosaad; N.Hanna; M.A. Moussa; M.A.Gouda; M.A. Salem; A. Khattab; A. Abdel-Latif;A. El-Hag; Najwa Abdel- Fattah; F. Hefnawy ;Mostafa, M. A.; M.S. Sharshar; T. Shehab El Din; M. Abde-Aleem; S.R.Sabry; *I. A. Imbaby and *M.M El- Shamy,(2013). Gemmeiza 11 A new Egyptian high yielding bread wheat (Triticum aestivum L.) cultivar, J.Agric. Sci., Mansoura Univ., 4(2):183-204.

Shehab El-Din T.; R.A. Mitkees.; M.M. El- Shamy; M.A. Gouda; M.M. AbdeAleem; A.M. Abdel- Ghani; N.S. Hanna; Iman M.M. Sadek; A.M. AboWarda; M.Kh. Mosherf; E.A.M. El-Sayed; Hayam S. Mahgoub; A.K.Mostafa; M.G. Mosaad; A.H. Bassiouni; M.M.A. El-Menofy; S.Kh. Mahmoud; M.A. Mahrous; A.A. Ageez; M.A.M Eid; M.H. Iskandar; M.A. Mostafa; A.A. Hamada; Y.G. Abdel- Gwad; A.M. Mousa; S.A. AbdelMajeed; A.M. Tammam; Nagwa R. Abdel- Fatah; H. Ashoush; F.A. Hefnawy; H. Hendawy; S.El-Din Ali; M.B. Toweefles; A.A. Abdel-Karim; A.A.Khattab; A.A.Gomaa;O.H.S.Khalill; Kadria F, Hegazi; Enayat H, Ghanem; A.A.Ali; F.F. El-Sayed; Ikhlas Safik and S. AboNaga,(1999):Sakha 93 and Giza 168:Two new high yielding and rust diseases resistant bread wheat cultivars. J. Agric. Sci., Mansoura Univ., 24:2157-2168.

Steel, R.G.D and Torrie, J.H. (1960): Principles and procedures of Statistics. $2^{\text {nd }}$ Ed. MC Graw-Hill Book Co. New York.

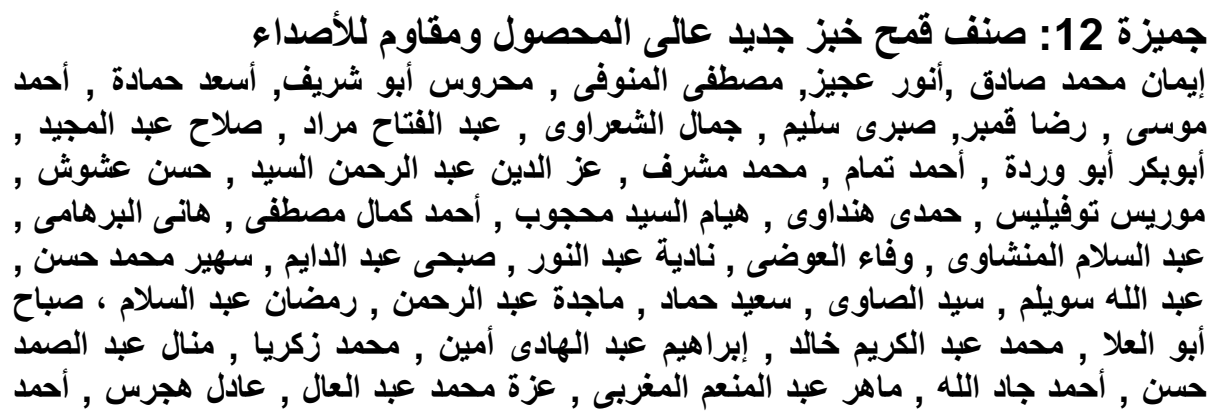




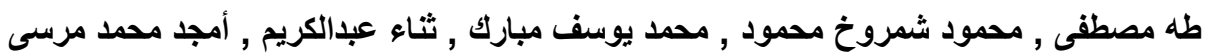

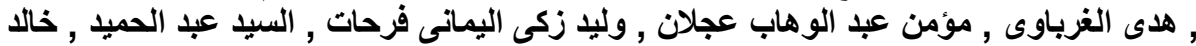

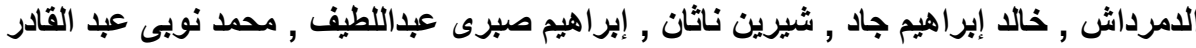

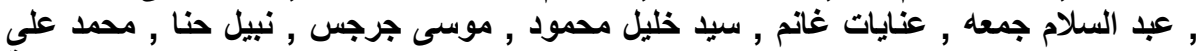

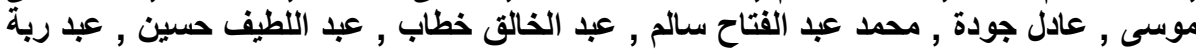

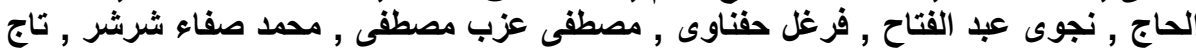

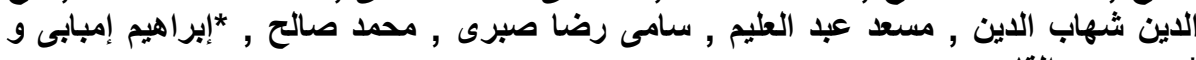

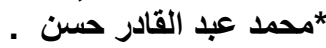

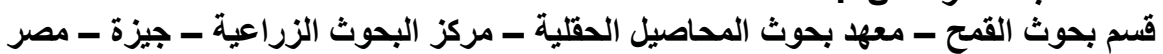

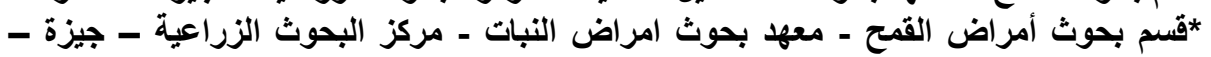

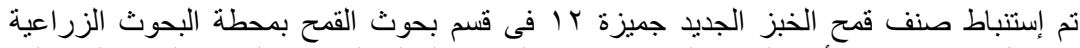

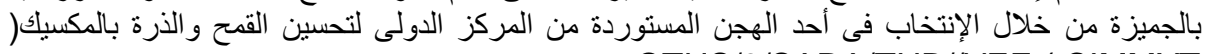
OTUS/3/SARA/THB//VEE ( CIMMYT

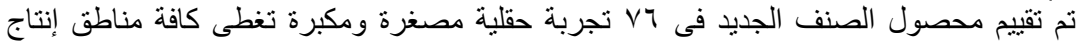

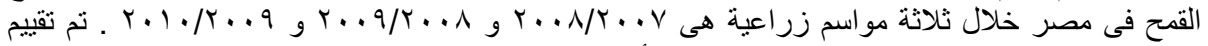

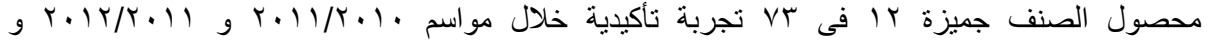

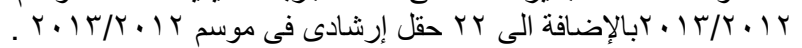

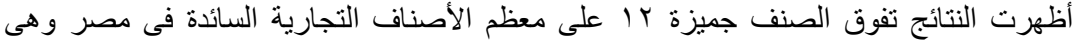

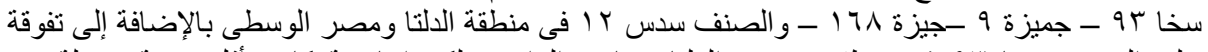

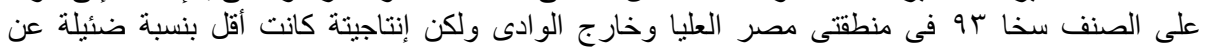

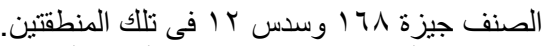

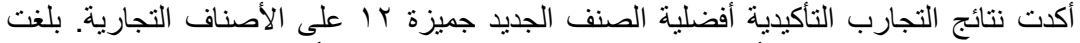

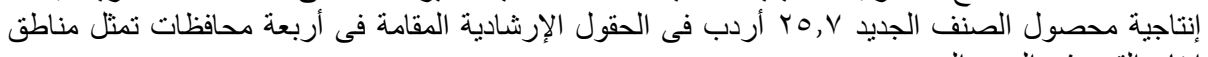

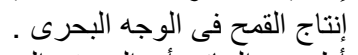

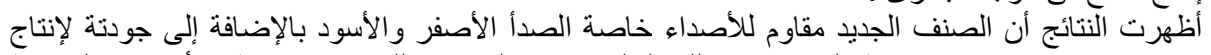

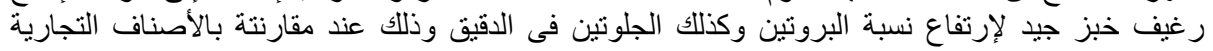

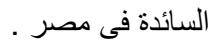

كلية الزراعة - جامعة المنصورة قام بتحكيم البحث كلية الزراعة ـ جامعة الزقازيق

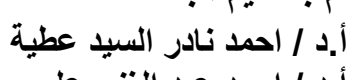
أ.د / احمد عبد الغنى على ندي الديل 